\title{
Supraglottic airways and pulmonary aspiration: the role of the drain tube
}

\author{
Pierre Drolet, MD
}

Published online: 27 August 2009

(c) Canadian Anesthesiologists' Society 2009

Several methods have already been proposed for the insertion of supraglottic devices. A study in this issue of the Journal describes a technique involving the prior insertion of a bougie into the upper esophagus over which the drain tube of the ProSeal ${ }^{\mathrm{TM}}$ laryngeal mask airway (PLMA) can be guided. Compared with the use of the metal introducer provided by the manufacturer, this method-with the drain tube-provides better positioning of the PLMA. ${ }^{1}$ It is worth noting that the authors concluded that the bougie technique is better because it allows alignment of the tip of the drain tube and the esophageal opening in a more predictable manner, not because the insertion time is shorter or the performance as a ventilatory device is improved. In fact, the bougie technique requires more time, more steps, and the use of a laryngoscope. Many clinicians will argue that the insertion strategy suggested by the authors of this study and others before them ${ }^{2}$ unnecessarily complicates a technique considered both simple and effective; therefore, they will likely be reluctant to adopt the technique on a routine basis. This is a paradoxical situation since many anesthesiologists view the incorporation of technical changes designed to prevent pulmonary aspiration to supraglottic devices as a significant improvement.

It is worth mentioning that developers of supraglottic airways have long been concerned about pulmonary aspiration and remain attentive to its prevention. The original laryngeal mask, now called Classic ${ }^{\mathrm{TM}}$, is equipped with an inflatable cuff that is designed not only to provide an adequate airway seal, but also to obstruct the esophagus in

P. Drolet, MD $(\square)$

Département d'anesthésiologie, Hôpital Maisonneuve-

Rosemont, Université de Montréal, 5415, boul. l'Assomption,

Montréal, QC H1T 2M4, Canada

e-mail: pdrolet@aei.ca order to reduce the risk of gastric contents reaching the laryngeal opening. Other devices also include an inflatable cuff (King-LT ${ }^{\mathrm{TM}}$ laryngeal tube) or feature components of various shapes and sizes (PAXpress ${ }^{\mathrm{TM}}$, CobraPLA ${ }^{\mathrm{TM}}$ ), all designed to occlude the upper esophagus. Another device, the SLIPA ${ }^{\mathrm{TM}}$ (Streamlined Liner of the Pharynx Airway), includes a reservoir designed to collect regurgitated gastric contents. ${ }^{3}$ In recent years, however, the most commonly observed tendency in the design of supraglottic airways is the incorporation of a lumen designed to drain secretions from the digestive tract. Some manufacturers achieved this by modifying the design of existing devices, thus resulting in the development of the PLMA, the Supreme ${ }^{\mathrm{TM}}$ laryngeal mask, or the King laryngeal tube (King LTS-D ${ }^{\mathrm{TM}}$ ). Others developed new devices, for instance, the $\mathrm{I}-\mathrm{Gel}^{\mathrm{TM}}$ or the EasyTube $^{\mathrm{TM}}$. This strong interest in supraglottic devices offering a separate lumen to the digestive tract even led some authors to assess the use of the venerable Combitube $^{\mathrm{TM}}$ for routine airway management during anesthesia. ${ }^{4}$

What evidence or arguments led manufacturers to opt for the integration or addition of a drain tube on their devices? We note that the approach is largely theoretical. Indeed, it is difficult to demonstrate that supraglottic airways without access to the digestive tract are responsible for a significant increase in pulmonary aspiration episodes during general anesthesia. Studies involving large patient populations, ${ }^{5,6}$ including some performed in populations traditionally considered at risk, such as Cesarean delivery patients, ${ }^{7}$ do not suggest that these devices are associated with a high incidence of clinically significant episodes of aspiration. Other studies involving indirect measures, such as pharyngeal $\mathrm{pH}^{8}$ or the use of dye (methylene blue), ${ }^{9}$ show that the separation provided by supraglottic devices between the digestive tract and the airway is not perfect. On the other hand, we have known for a long time that 
tracheal intubation, when studied with these markers, does not offer absolute protection against airway contamination either. ${ }^{10,11}$ In a few studies performed on fresh cadavers, the authors attempted to assess the level of protection offered by supraglottic devices when confronted with an increase in gastric contents and esophageal pressure. These studies showed that devices obstructing the esophagus, but not equipped with a drain tube, are generally resistant to mean pressures approaching or greater than $40 \mathrm{~cm} \mathrm{H}_{2} \mathrm{O}$ before gastric contents are released and reach the airway. ${ }^{12,13}$ Since its shape did not allow for appropriate obstruction of the esophagus, the COPA ${ }^{\mathrm{TM}}$ (Cuffed Oropharyngeal Airway), one of the oldest airways, was the only device proven to be significantly less effective. ${ }^{14}$ Of course, the overall incidence of clinically significant pulmonary aspiration episodes associated with general anesthesia remains low, making it unlikely that a randomized controlled trial of sufficient size to address the issue of pulmonary aspiration with tracheal intubation versus supraglottic devices will ever be conducted.

Of course, this does not mean that the addition or incorporation of an esophageal drain tube to a supraglottic airway device is useless. When confronted with rare problems that can have major consequences, sometimes measures must be taken based on sound physiological or pathophysiological principles, knowing that it will be difficult to gather convincing epidemiological evidence regarding the issue. Capnometry and pulse oximetry won recognition as common monitoring tools on this basis, despite the lack of evidence regarding their impact on outcomes for surgical patients. Nevertheless, there are published case reports that seemingly support the fact that devices equipped with drain tubes can improve patient safety. These reports mention fluids, sometimes in large quantity, that are emitted from the digestive tract and evacuated via the drain tube. ${ }^{15,16}$ These cases are obviously anecdotal, nevertheless, they provide an argument for the usefulness of such a tube. Studies performed on fresh cadavers also lead us to believe that the drain tube can reduce the likelihood of pulmonary aspiration. Indeed, when a supraglottic airway is adequately positioned and its drain tube is aligned correctly with the esophagus, it becomes very difficult to generate esophageal pressures sufficient to create leaks toward the airway. Even when the drain tube is obstructed, it is worth noting that the devices equipped with such a tube can generally resist esophageal pressures equal to or greater than those observed with devices without a drain tube. Therefore, it is still possible that these devices offer a significant level of protection against regurgitation, even if the drain tube is inadequately positioned and not in line with the esophagus. Only the I-Gel ${ }^{\mathrm{TM}}$, which does not have an inflatable cuff, appears to allow gastric contents to leak at a median esophageal pressure lower than $20 \mathrm{~cm}$ $\mathrm{H}_{2} \mathrm{O}$ when the drain tube is obstructed. ${ }^{17}$

Although the drain tube is generally viewed as a feature likely to increase patient safety, it still raises a few concerns. First, let us emphasize that passage of gastric contents into the airway remains a possibility despite the presence of a drain tube; such a case was reported with the I$\mathrm{Gel}^{\mathrm{TM}}{ }^{18}$ Obviously, the drain tube must be aligned with the esophagus in order to be functional. Incidentally, even though several authors mention the presence of a drain tube as a significant feature to justify studying a given supraglottic device, it is interesting to note that they often fail to check its effectiveness or adequate positioning. In many articles, only the ventilatory performance of the devices was studied and compared. A device can be absolutely functional from a respiratory viewpoint even if the drain opening is inadequately positioned. In some studies, it is mentioned that a gastric tube was inserted trough the drain; however, even when such insertion is successful, it sometimes requires efforts that suggest that the alignment of the structures was sub-optimal and therefore potentially ineffective in case of regurgitation. In their study, El Beheiry et al. suggest that $20 \%$ of PLMAs inserted with the metal introducer provided by the manufacturer do not result in optimal alignment between the drain tube and the esophageal opening. Their method, fibroscopic observation of the esophageal mucosa, is probably more apt to detect slightly improper alignment than the insertion of a gastric tube that can be pushed forcefully down the drain toward the esophagus without concern for breaking expensive material. Therefore, it will be interesting to see whether future researchers also select this particular evaluation technique.

Many clinicians consider the PLMA as being more difficult to insert than the Classic ${ }^{\mathrm{TM}}$ laryngeal mask. ${ }^{19,20}$ Despite this difficulty, they prefer the PLMA because of its drain tube and its potential role in preventing pulmonary aspiration. If the drain proves ineffective in one out of five patients, consequently, a significant number of patients do not benefit from the desired extra protection. It is difficult to measure the real impact of this observation on patient safety since, as previously mentioned, clinically significant pulmonary aspiration is a rare event, and even if supraglottic airways equipped with a drain tube do not provide a perfect alignment with the digestive tract, they can still obstruct the esophagus and offer some protection against regurgitation. Nevertheless, when a device with a drain tube is chosen, it is because the clinician wishes to provide the patient with its potential benefits. This raises several questions: Should we recommend the routine use of more complex insertion techniques, such as the one suggested by the authors, to ensure the drain tube does its job? Which one, among the various techniques described for PLMA 
insertion, is most likely to ensure adequate positioning of the drain tube? For instance, some authors have recommended using a gastric tube that protrudes from the tip of the drain tube to serve as a guide during digital insertion. ${ }^{21}$ Others have advocated inserting a rigid stylet into the drain tube to prevent the tip of the supraglottic airway from folding on itself. ${ }^{22}$ Should we use these particular techniques only in situations where the concern for gastroesophageal reflux is greatest, as in the case of obese patients or laparoscopic surgery? Doesn't such an approach strip supraglottic airways of some of the simplicity that distinguishes them from tracheal intubation? Are some supraglottic airways more likely to provide a functional drain, notwithstanding the insertion technique? Of course, one should also bear in mind that preventing pulmonary aspiration is only one among the many desired characteristics of supraglottic airways. Ventilatory performance and absence of other side effects (sore throat, trauma, etc.) are also crucial elements that must be taken into account when choosing an airway device and an insertion technique.

In conclusion, several supraglottic airways are now equipped with drain tubes or other mechanisms specifically designed to prevent pulmonary aspiration. Although their true impact on patient safety is not yet clear, clinicians appear to have adopted these new designs. Therefore, it would seem appropriate that future clinical research conducted on new supraglottic airways, their use, and insertion techniques routinely integrates measures to test if the component designed to prevent aspiration, particularly the drain tube, are properly positioned. Such information will provide clinicians with a more complete picture of the performance of these devices and help them choose which one, and which insertion technique, are best suited for each patient's needs.

\section{Les instruments ventilatoires supraglottiques et l'inhalation pulmonaire: le rôle du tube de drainage}

Plusieurs méthodes d'insertion des outils supraglottiques ont déjà été proposées. Dans ce numéro du Journal, une étude décrit une technique impliquant l'insertion préalable d'une bougie dans l'oesophage supérieur sur laquelle on glisse le tube de drainage du masque laryngé de type ProSeal $^{\mathrm{TM}}$ (PLMA). Cette méthode amène un meilleur positionnement de ce dernier lorsque comparée à l'utilisation de l'outil métallique proposé par le fabricant. ${ }^{1}$ Il est intéressant de constater que les auteurs concluent à la supériorité de la technique utilisant la bougie, non pas parce qu'elle rend l'insertion plus rapide ou que la performance ventilatoire est meilleure, mais plutôt parce qu'elle permet d'aligner l'orifice du tube de drainage et l'ouverture oesophagienne de manière plus prévisible. En fait, la technique sur bougie requiert plus de temps, plus d'étapes, et fait appel à l'utilisation d'un laryngoscope. Il est donc probable que plusieurs cliniciens hésiteront à adopter de manière systématique la stratégie d'insertion proposée par les auteurs de l'étude et par d'autres avant eux, ${ }^{2}$ leur reprochant de complexifier inutilement une technique perçue comme simple et efficace. Il s'agit là d'une situation qui peut sembler paradoxale puisque, aux yeux de plusieurs anesthésiologistes, l'incorporation aux instruments de ventilation supraglottiques de modifications techniques visant à protéger le malade de l'inhalation pulmonaire représente une amélioration significative dans l'évolution de ce type d'outils.

Il convient de mentionner que la crainte de l'inhalation pulmonaire et sa prévention sont des préoccupations qui ne sont pas nouvelles pour les concepteurs d'appareils ventilatoires supraglottiques. Le masque laryngé original, maintenant appelé Classic ${ }^{\mathrm{TM}}$, est muni d'une manchette gonflable destinée non seulement à assurer une étanchéité ventilatoire, mais aussi à obstruer l'œsophage afin de réduire la possibilité que le contenu gastrique puisse atteindre l'orifice laryngé. Plusieurs autres instruments incorporent également des éléments destinés à obstruer l'ouverture oesophagienne, qu'il s'agisse de manchettes gonflables (tube laryngé King-LT ${ }^{\mathrm{TM}}$ ) ou de structures dont la forme et le volume varient selon les modèles (PAXpress $^{\mathrm{TM}}$, CobraPLA ${ }^{\mathrm{TM}}$ ). Un autre appareil appelé SLIPA ${ }^{\mathrm{TM}}$ (Streamlined Liner of the Pharynx Airway) mise plutôt sur la présence d'un réservoir destiné à recueillir le matériel gastrique régurgité. ${ }^{3}$ La tendance la plus importante observée au cours des dernières années consiste cependant à incorporer aux instruments de ventilation supraglottiques une lumière destinée au drainage des sécrétions émanant du tube digestif. Certains fabricants y sont parvenus en apportant des modifications conceptuelles à des appareils déjà existants, qu'il s'agisse du PLMA, du masque laryngé de type Supreme ${ }^{\mathrm{TM}}$ ou du tube laryngé King (King LTS$\left.\mathrm{D}^{\mathrm{TM}}\right)$. D'autres manufacturiers ont proposé de nouveaux outils tels que le $\mathrm{I}_{-} \mathrm{Gel}^{\mathrm{TM}}$ ou le EasyTube ${ }^{\mathrm{TM}}$. Cet engouement pour les appareils supraglottiques offrant un passage séparé vers le tube digestif a même amené certains auteurs à évaluer l'utilisation du vénérable Combitube ${ }^{\mathrm{TM}}$ pour la prise en charge routinière des voies aériennes durant l'anesthésie. ${ }^{4}$

Quels sont les données probantes ou arguments qui ont amené les fabricants à opter pour l'intégration ou l'ajout d'un tube de drainage sur ces appareils? Nous constatons que l'approche est souvent de nature empirique. Il est en effet difficile de démontrer que les outils de ventilation 
supraglottiques n'offrant pas d'accès au tube digestif sont responsables d'une hausse significative des syndromes d'inhalation pulmonaire durant l'anesthésie générale. Des études impliquant un grand nombre de patients, ${ }^{5,6}$ dont certaines réalisées chez des clientèles traditionnellement perçues comme vulnérables telles que les patientes subissant des césariennes, ${ }^{7}$ ne suggèrent pas que ces appareils soient associés à une incidence élevée d'épisodes d'inhalation cliniquement significatifs. D'autres études ayant recours à des mesures indirectes comme le $\mathrm{pH}$ pharyngé ${ }^{8}$ ou l'utilisation de colorant (bleu de méthylène) ${ }^{9}$ montrent que le cloisonnement entre le tractus digestif et les voies respiratoires offert par les outils supraglottiques n'est pas total. Nous savons cependant depuis longtemps que l'intubation trachéale n'offre pas non plus une protection absolue lorsque étudiée avec ce type de marqueurs. ${ }^{10,11}$ Quelques travaux menés sur des cadavres frais ont tenté d'estimer la protection offerte par les appareils supraglottiques face à l'augmentation du contenu gastrique et de la pression oesophagienne. Ces travaux ont montré que les appareils obstruant l'œsophage, mais non munis d'un tube de drainage, résistent généralement à des pressions moyennes avoisinant ou dépassant $40 \mathrm{~cm} \mathrm{H}_{2} \mathrm{O}$ avant de laisser échapper le contenu digestif et de lui permettre de rejoindre les voies respiratoires. ${ }^{12,13}$ Seul le COPA $^{\mathrm{TM}}$ (Cuffed Oropharyngeal Airway), un instrument supraglottique maintenant pratiquement disparu et dont la forme ne permettait pas d'obstruer convenablement l'œsophage, s'avérait significativement moins efficace. ${ }^{14}$ Évidemment, l'incidence des épisodes d'inhalation pulmonaire cliniquement significatifs associés à l'anesthésie générale demeure faible. Il semble donc peu probable qu'une étude prospective randomisée de taille suffisante pour comparer l'impact de l'intubation trachéale et des appareils supraglottiques sur l'incidence de cette complication puisse être mise sur pied.

Cela ne signifie évidemment pas que l'ajout ou l'intégration d'un tube visant le drainage digestif aux instruments de ventilation supraglottiques soit une mesure futile. Lorsque confronté à des problèmes rares mais dont les conséquences peuvent être majeures, il faut parfois se résoudre à adopter des mesures qui reposent sur des principes physiologiques ou physiopathologiques solides, même en sachant que les preuves scientifiques sur le sujet seront difficiles à recueillir, en particulier sur le plan épidémiologique. C'est ainsi que la capnométrie et l'oxymétrie de pouls se sont imposées comme des outils de monitorage courants, et ce, même en l'absence de données probantes quant à leur impact sur le pronostic des patients opérés. Il existe tout de même des cas publiés qui laissent croire qu'effectivement, les appareils munis d'un tube de drainage sont en mesure d'améliorer la sécurité des patients. Ces rapports font mention de liquide, parfois en quantité importante, provenant du tractus digestif et qui est expulsé via le tube de drainage. ${ }^{15,16}$ Évidemment, il s'agit là de cas anecdotiques, mais qui militent néanmoins en faveur de la présence d'un tel tube. Les études menées chez les cadavres frais permettent aussi de croire que le tube de drainage est en mesure de réduire la probabilité d'inhalation pulmonaire. En effet, lorsqu'un appareil ventilatoire supraglottique est convenablement installé et que son tube de drainage communique directement avec l'œsophage, il devient très difficile de générer des pressions oesophagiennes suffisantes pour entraîner des fuites vers les voies respiratoires. Il est intéressant de noter que même lorsque leur tube de drainage est obstrué, les instruments qui en sont munis sont généralement capables de résister à des pressions oesophagiennes égales ou supérieures à celles observées sur les appareils sans tube de drainage. Il est donc possible que ces outils offrent tout de même un degré de protection significatif contre les régurgitations, et ce, même si le tube de drainage est mal placé et ne communique pas avec l'œsophage. Seul le $\mathrm{I}-\mathrm{Gel}^{\mathrm{TM}}$, un appareil non muni d'une manchette gonflable, semble permettre au contenu digestif de fuir à une pression oesophagienne médiane inférieure à $20 \mathrm{~cm} \mathrm{H}_{2} \mathrm{O}$ lorsque son tube de drainage est obstrué. ${ }^{17}$

Si le tube de drainage est généralement perçu comme un élément susceptible d'accroître la sécurité des patients, sa présence suscite néanmoins quelques réflexions. Soulignons d'abord que malgré la présence d'un tel tube, le passage de liquide gastrique vers les voies respiratoires demeure possible, un tel cas ayant été rapporté avec le $\mathrm{I}-\mathrm{Gel}^{\mathrm{TM}} \cdot{ }^{18}$ Évidemment, pour être fonctionnel, le tube de drainage doit faire face à l'œsophage. Il est d'ailleurs intéressant de constater que même si plusieurs auteurs mentionnent souvent la présence d'un tube de drainage comme une caractéristique importante afin de justifier leur intérêt pour l'étude d'un instrument supraglottique donné, ils négligent souvent d'en vérifier l'efficacité ou le bon positionnement. Dans un grand nombre d'études, seules les caractéristiques ventilatoires des instruments font l'objet d'observations et de comparaisons. Ce type d'appareil peut s'avérer parfaitement fonctionnel au plan respiratoire, alors que l'orifice du drain est en position inadéquate. Certains travaux de recherche font mention de l'insertion d'un tube gastrique dans le drain de l'appareil étudié, mais même lorsque celle-ci s'avère fructueuse, elle nécessite parfois des efforts qui laissent supposer que l'alignement des structures était sous-optimal et donc potentiellement inefficace en cas de régurgitations. L'étude de El Beheiry et coll. suggère que $20 \%$ des PLMA insérés à l'aide de l'outil métallique fourni par le fabricant n'offrent pas un alignement optimal du tube de drainage et de l'ouverture oesophagienne. La méthode utilisée, soit l'observation fibroscopique de la muqueuse digestive, est probablement 
plus sensible que l'insertion d'un tube gastrique sur lequel une pression importante peut être exercée sans crainte de bris matériel. Il sera donc intéressant de constater si d'autres auteurs auront recours à cette technique d'évaluation afin d'en préciser la valeur.

Le PLMA est considéré par plusieurs comme étant plus difficile à insérer que le masque laryngé Classic ${ }^{\mathrm{TM}} \cdot{ }^{19,20}$ Malgré cela, beaucoup de cliniciens le préfèrent en raison de la présence du tube de drainage et de son rôle potentiel dans la prévention de l'inhalation pulmonaire. Si le drain s'avère inefficace chez un patient sur cinq, cela indique qu'un nombre significatif de patients ne bénéficient pas de cette protection supplémentaire pourtant recherchée. Il est difficile de mesurer l'impact réel d'une telle observation sur la sécurité des patients puisque tel que mentionné précédemment, l'inhalation pulmonaire cliniquement significative est rare et que les outils supraglottiques munis d'un tube de drainage, même si ce dernier est non perméable, peuvent néanmoins obstruer l'œsophage et offrir ainsi une certaine protection contre les régurgitations. Il demeure tout de même que si le clinicien opte pour un instrument muni d'un drain, c'est qu'il souhaite procurer au patient les avantages potentiels de ce dernier. Cela soulève plusieurs questions : Faut-il, pour s'assurer du bon fonctionnement du tube de drainage, encourager l'utilisation systématique de techniques d'insertion plus complexes telles que celle proposée par les auteurs? Laquelle, parmi les techniques d'insertion décrites pour l'insertion du PLMA, est la plus susceptible d'assurer un positionnement adéquat du tube de drainage? Par exemple, certains auteurs ont préconisé, durant l'insertion digitale, l'utilisation d'un tube gastrique dépassant l'extrémité du tube de drainage afin d'agir comme guide ${ }^{21}$ tandis que d'autres favorisent l'insertion d'un stylet rigide dans le tube de drainage afin d'éviter que l'extrémité de l'outil supraglottique ne puisse se replier $^{22} \ldots$ Faut-il réserver ce type de technique aux situations pour lesquelles la crainte du reflux gastrooesophagien est plus grande, comme dans les cas de patients obèses ou de chirurgie laparoscopique? Une telle approche n'enlève-t-elle pas aux instruments supraglottiques certains éléments de simplicité qui les distinguent de l'intubation trachéale? Certains appareils supraglottiques sont-ils plus susceptibles de procurer un drain fonctionnel, nonobstant la technique d'insertion? Il ne faut évidemment pas oublier que la prévention des inhalations pulmonaires ne représente qu'une seule des caractéristiques recherchées chez les appareils supraglottiques. La performance ventilatoire et l'absence d'autres effets indésirables (maux de gorge, traumatismes, etc.) sont aussi des éléments essentiels qui doivent être considérés dans le choix de l'instrument et de sa technique d'insertion.

En conclusion, plusieurs appareils supraglottiques sont maintenant dotés de tubes de drainage ou d'autres mécanismes destinés spécifiquement à la prévention des épisodes d'inhalation pulmonaire. Bien que leur impact réel sur la sécurité des patients ne soit pas clairement défini, les cliniciens semblent néanmoins avoir adopté ces nouveaux concepts. Il faut donc souhaiter que les recherches cliniques futures portant sur les nouveaux instruments supraglottiques, ou sur la manière de les utiliser et de les insérer, incorporent systématiquement un volet dont le but sera de tester l'aspect fonctionnel des mesures destinées à la prévention des inhalations, en particulier en ce qui a trait au bon positionnement des tubes de drainage. Les informations ainsi recueillies permettront au clinicien d'obtenir une image plus complète de la performance de ces instruments et ainsi d'opter pour l'appareil ou la technique d'insertion la plus susceptible de tenir compte des besoins spécifiques de chaque patient.

Conflicts of interest None declared.

\section{References}

1. El Beheiry H, Wong J, Nair G, et al. Improved esophageal patency when inserting the ProSeal ${ }^{\mathrm{TM}}$ Laryngeal Mask Airway with an Eschmann ${ }^{\mathrm{TM}}$ tracheal tube introducer. Can J Anesth 2009. doi: 10.1007/s12630-009-9141-7.

2. Brimacombe J, Keller C, Judd DV. Gum elastic bougie-guided insertion of the ProSeal laryngeal mask airway is superior to the digital and introducer tool techniques. Anesthesiology 2004; 100: 25-9.

3. Miller DM, Light D. Laboratory and clinical comparisons of the Streamlined Liner of the Pharynx Airway (SLIPA) with the laryngeal mask airway. Anaesthesia 2003; 58: 136-42.

4. Gaitini LA, Vaida SJ, Mostafa S, et al. The Combitube in elective surgery: a report of 200 cases. Anesthesiology 2001; 94: 79-82.

5. Verghese C, Brimacombe JR. Survey of laryngeal mask airway usage in 11,910 patients: safety and efficacy for conventional and nonconventional usage. Anesth Analg 1996; 82: 129-33.

6. Verghese C, Smith TG, Young E. Prospective survey of the use of the laryngeal mask airway in 2359 patients. Anaesthesia 1993; 48: 58-60.

7. Han TH, Brimacombe J, Lee EJ, Yang HS. The laryngeal mask airway is effective (and probably safe) in selected healthy parturients for elective cesarean section: a prospective study of 1067 cases. Can J Anesth 2001; 48: 1117-21.

8. Hagberg CA, Vartazarian TN, Chelly JE, Ovassapian A. The incidence of gastroesophageal reflux and tracheal aspiration detected with $\mathrm{pH}$ electrodes is similar with the Laryngeal Mask Airway and Esophageal Tracheal Combitube-a pilot study. Can J Anesth 2004; 51: 243-9.

9. Lallo A, Drolet P, Lacroix M. Performance of the PAxpress vs the ProSeal laryngeal mask airway during general anesthesia. Can J Anesth 2007; 54: 28-33.

10. Petring OU, Adelhoj B, Jensen BN, Pedersen NO, Lomholt $N$. Prevention of silent aspiration due to leaks around cuffs of endotracheal tubes. Anesth Analg 1986; 65: 777-80.

11. Russell GN, Yam PC, Tran J, et al. Gastroesophageal reflux and tracheobronchial contamination after cardiac surgery: should a nasogastric tube be routine? Anesth Analg 1996; 83: 228-32. 
12. Bercker S, Schmidbauer W, Volk T. A comparison of seal in seven supraglottic airway devices using a cadaver model of elevated esophageal pressure. Anesth Analg 2008; 106: 445-8.

13. Keller C, Brimacombe J, Kleinsasser A, Loeckinger A. Does the ProSeal laryngeal mask airway prevent aspiration of regurgitated fluid? Anesth Analg 2000; 91: 1017-20.

14. Brimacombe J, Keller C. Water flow between the upper esophagus and pharynx for the LMA and COPA in fresh cadavers. Laryngeal mask airway, and cuffed oropharyngeal airway. Can J Anesth 1999; 46: 1064-6.

15. Su BC, Yang MW, Lee HC, Chang CH, Lin CC. Protection against large-volume regurgitated fluid aspiration by the ProSeal laryngeal mask airway. Acta Anaesthesiol Taiwan 2008; 46: 34-8.

16. Evans NR, Llewellyn RL, Gardner $S V$, James $M F$. Aspiration prevented by the ProSeal laryngeal mask airway: a case report. Can J Anesth 2002; 49: 413-6.

17. Schmidbauer W, Bercker S, Volk T, Boqusch G, Mager G, Kerner T. Oesophageal seal of the novel supralaryngeal airway device I-Gel in comparison with the laryngeal mask airways Classic and ProSeal using a cadaver model. Br J Anaesth 2009; 102: 135-9.

18. Gibbison B, Cook TM, Seller C. Case series: protection from aspiration and failure of protection from aspiration with the i-gel airway. Br J Anaesth 2008; 100: 415-7.

19. Brimacombe J, Keller C, Fullekrug B, et al. A multicenter study comparing the ProSeal and Classic laryngeal mask airway in anesthetized, nonparalyzed patients. Anesthesiology 2002; 96: 289-95.

20. Cook TM, Nolan JP, Verghese C, et al. Randomized crossover comparison of the proseal with the classic laryngeal mask airway in unparalysed anaesthetized patients. Br J Anaesth 2002; 88: 527-33.

21. Drolet P, Girard M. An aid to correct positioning of the ProSeal laryngeal mask. Can J Anesth 2001; 48: 718-9.

22. Chen HS, Liu PH, Chung KC. The Rusch Flexi-Slip stylet for ProSeal laryngeal mask airway insertion. Can J Anesth 2008; 55 : 719-20. 